\title{
Review Paper \\ Caregiving Appraisal in Family Caregivers of Older Adults
}

\author{
Akram Farhadi ${ }^{1}$, Mahshid Foroughan $^{1}$, Farahnaz Mohammadi ${ }^{1}$, Maryam Rassouli ${ }^{2}$, Leila Sadegh Moghadam ${ }^{3}$, \\ Shima Nazari ${ }^{1}$, Narjes Sadeghi ${ }^{1}$
}

1. Iranian Research Centre on Ageing, University of Social Welfare \& Rehabilitation Sciences, Tehran, Iran.

2. Department of Nursing, Faculty of Nursing and Midwifery, Shahid Beheshti University of Medical Sciences, Tehran, Iran.

3. Department of Community and Mental Health Nursing, Faculty of Nursing and Midwifery, Gonabad University of Medical Sciences, Gonabad, Iran.

Received: 04 Dec. 2015 Accepted: 18 Feb. 2016

Key words: Elderly, Selfappraisal, Family caregivers, Review literature

\section{A B S TR A C T}

Objectives With increasing the older adults' population, the old age diseases increase, as well as older people's dependency and need for care. This issue further highlights the importance of attention to family caregiving and experiences. Among the concepts related to family caregiving, the concept of selfappraisal of caregiver has a special role in acceptance, continuance, and outcomes of caregiving. This study aimed to review all aspects of caregiving appraisal concept in family caregivers.

Methods \& Materials The current study is a review study. To examine the concept of family caregiver appraisal, all articles without time limitation were searched in databases of Google Scholar, ProQuest, Scopus, and PubMed with keywords of "appraisal," "caregiving," "family caregiving," "burden," "satisfaction," "positive aspect," "negative aspect," "older adults," and "elderly." The Iranian databases of IranMedex, IranDoc, and SID were also searched with equivalent Persian Keywords.

Results Many factors such as social, cultural, and personal characteristics can influence the caregivers' perception. This subjective appraisal makes caregivers not to perceive their conditions the same, in spite of stressful situation of caregiving. Some caregivers have positive experiences in this condition and some perceive stress more than what actually exist. This will create negative outcomes for the health and welfare of the caregivers.

Conclusion Caregiving appraisal is a multidimensional concept, which has positive and negative aspects. By taking into account all aspects of this concept, one can better understand and ultimately has a more comprehensive assessment of the status of caregivers, and implement effective interventions towards improving the health of this group.

\section{* Corresponding Author:}

Address: Iranian Research Centre on Ageing, University of Social Welfare and Rehabilitation Sciences, Kodakyar Ave., Daneshjo Blv., Evin, Tehran, Iran. Tel: +98 (21) 2280004

E-mail: m_foroughan@yahoo.com 


\title{
مقاله مرورى: مفهوم ارزيابى مراقبت در مراقبان خانوادتى سالمندان
}

اكرم فرهادى'، "مهشيد فروغان'، فرحناز محمدى'، مريم رسولى'، ليلا صادقمقدم"، شيما نظرى'، نرجسخاتون صادقى'

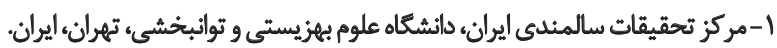

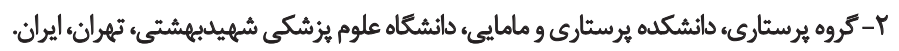

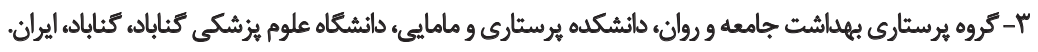

\begin{abstract}
حكSد

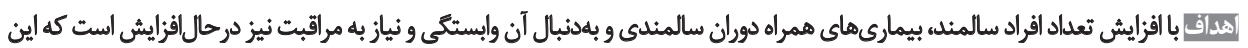

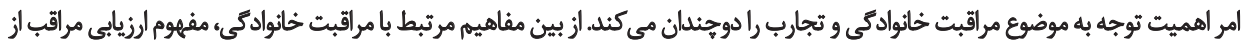

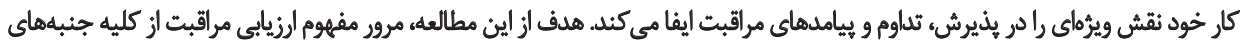

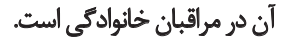

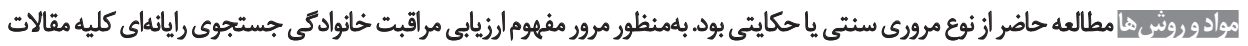

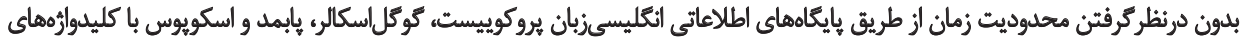
Appraisal, Caregiving, Family caregiving, Burden, Satisfaction, Positive aspect, Negative aspect, Older adults, Elderly

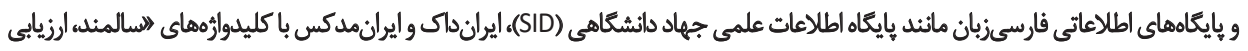

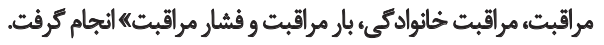

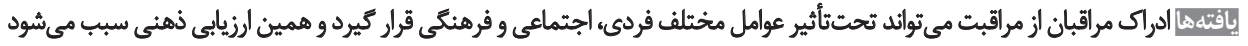

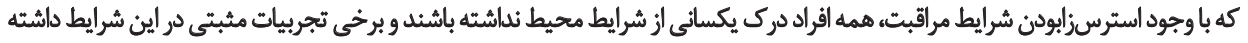

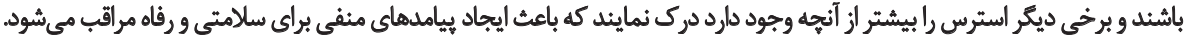

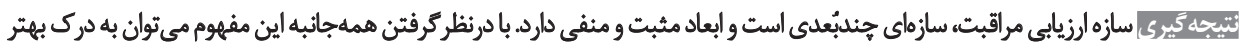

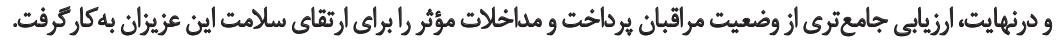

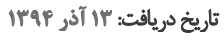

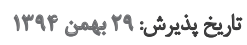

كليدوازهذها:

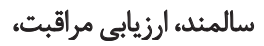

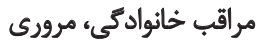

داشت؛ بيمارىهايى كه نهتنها فرد بلكه مراقبان، خانواده و كل

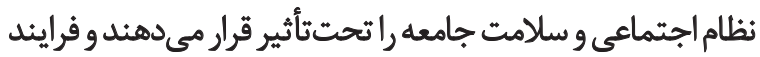

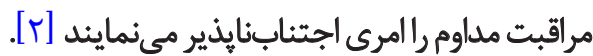

در سراسر دنيا، بهطور اعم در كشورهاى درحالتوسعه و وإنيت

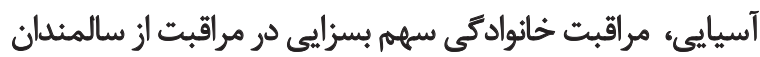

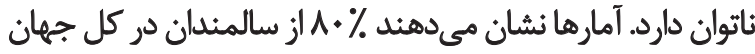

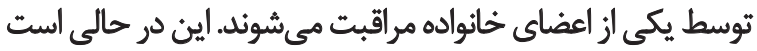

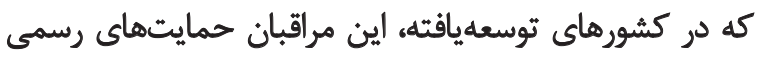

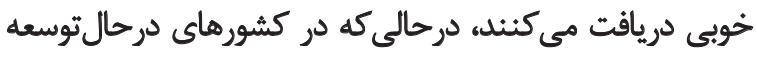

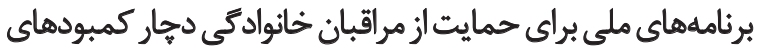

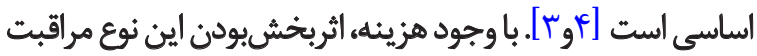

با افزايش سالانه اميدبلزندكى و كاهش مواليد، شاهد افزايش

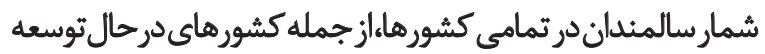

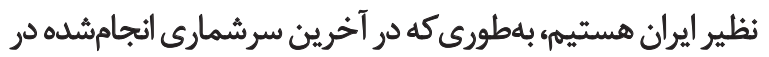

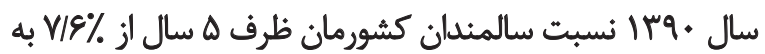

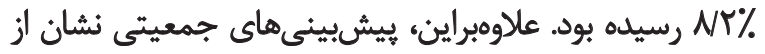

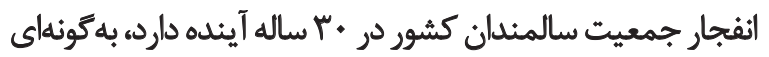

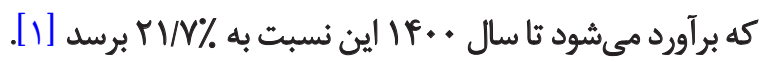

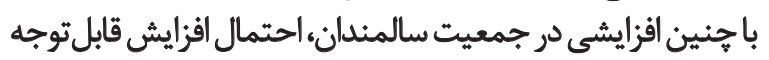

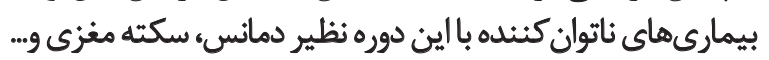

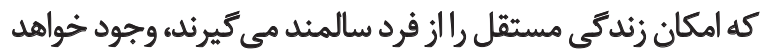




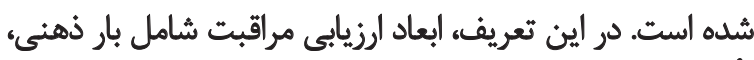

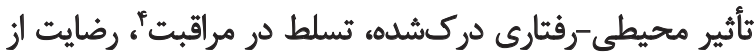
مراقب هو بازارزيابى است [11] [1]. بلهور كلى، يرؤششها نشان مي دهند كه از نظر مفاهيم در

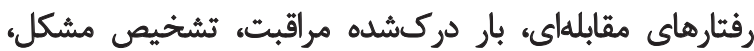

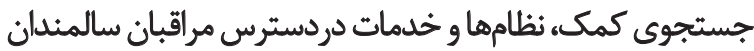

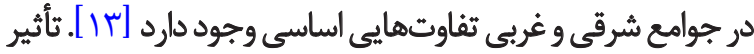

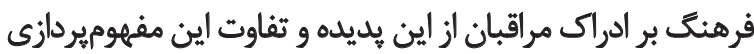

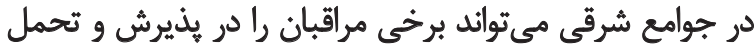

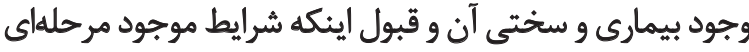

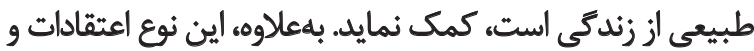

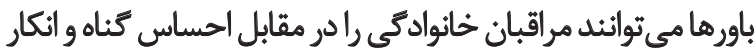

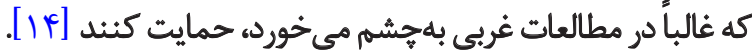

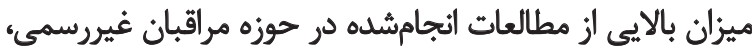

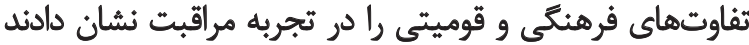

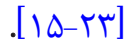

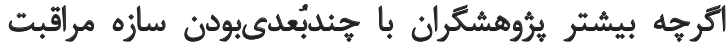

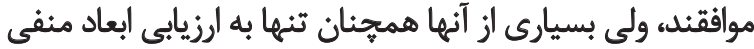

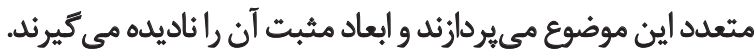

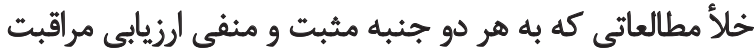

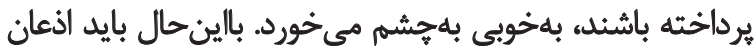

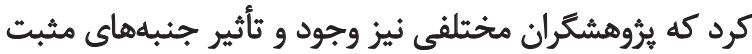

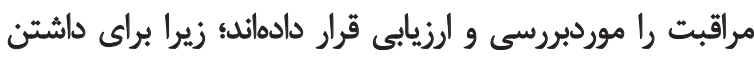

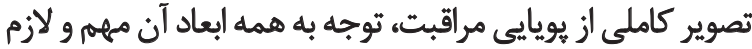

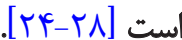

فهم هرجه بهتر مفاهيم مرتبط با تجربه مراقبت نظير ارزيابي

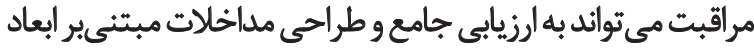

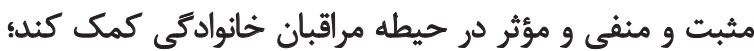

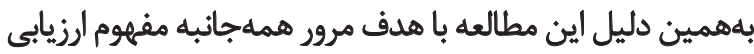

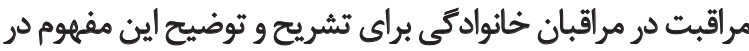

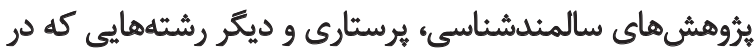
اين زمينه تلاش مى كننده انجام گرفت

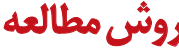

مطالعه حاضر از نوع مرورى سنتى ياحكايتى \$است كه بهمنظور

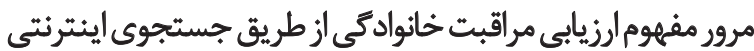

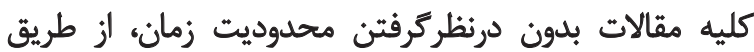

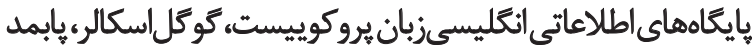

\footnotetext{
4. Caregiving mastery

5. Caregiving satisfaction

6. Narrative review
}

و اتكاى بيشاز حد به خانوادهها بدون كمك جدى بـ به آنهاء سبب

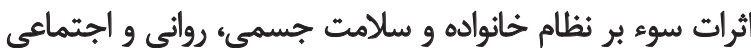

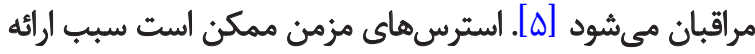

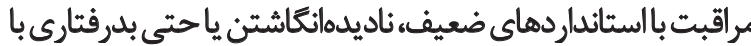

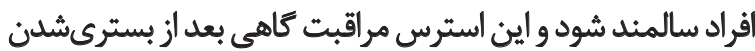

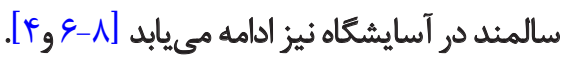
مراقبت خانوادكى يديده جديدى نيست و همواره در طول

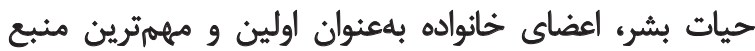

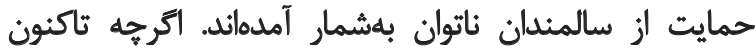

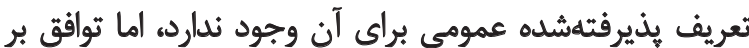

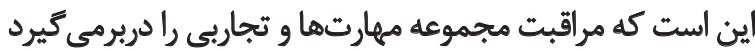

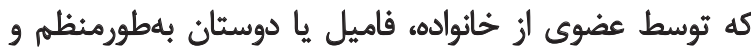

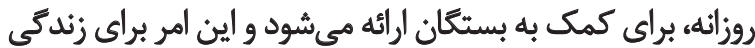

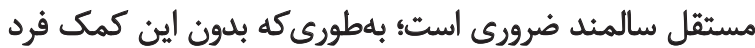

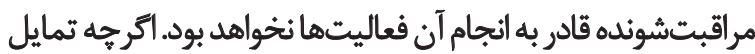

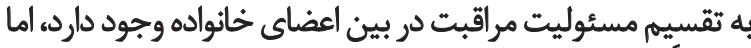

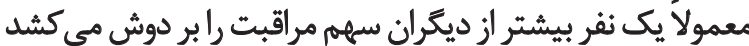

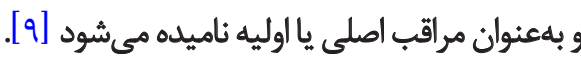
زمانى كه مراقب مسئوليت مراقبت رابرعهده مي كيرد، مفيدترين

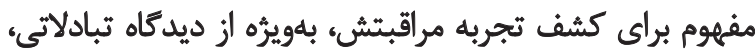

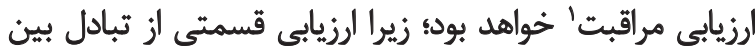

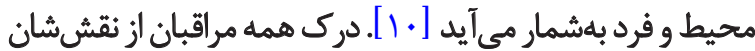

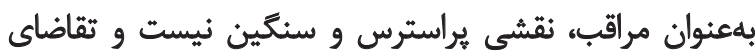

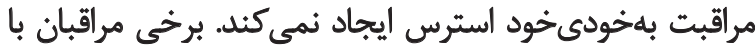

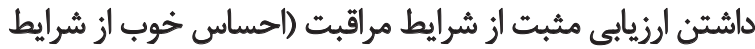

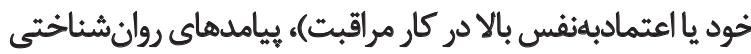

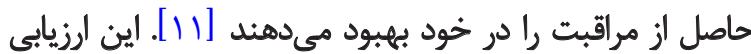

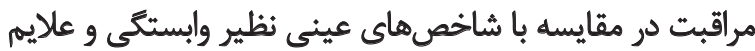

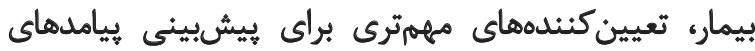

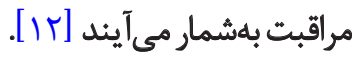

در بيشتر مطالعات انجامشده در زمينه ارزيابى مراقبان

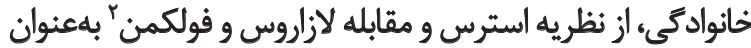

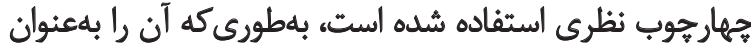

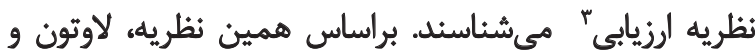

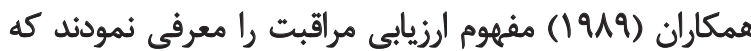

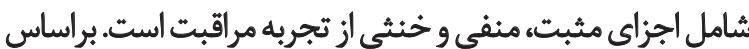

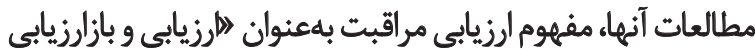

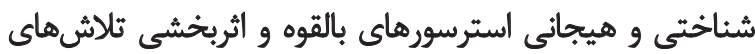
مقابلهاى مراقب در حين ارائه مراقبت به افراد سالمنده تعريف

1. Caregiving appraisal

2. Lazarus and Folkman

3. Appraisal theory 


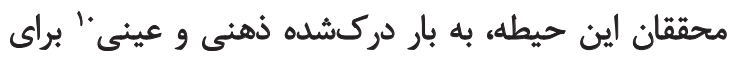

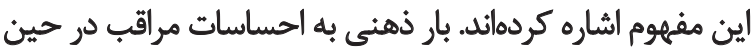

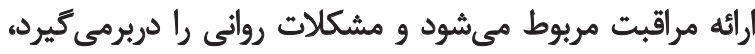

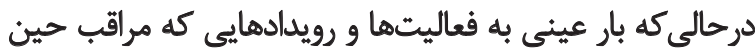

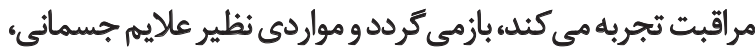

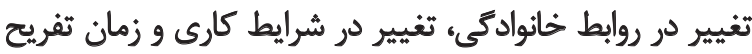

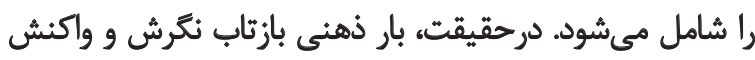

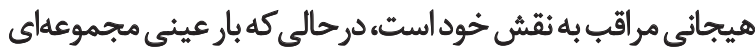

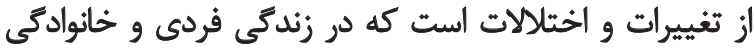

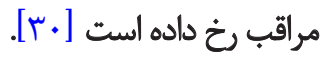

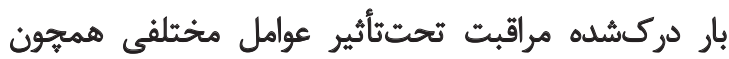

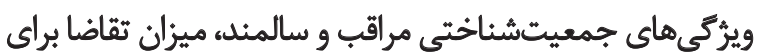

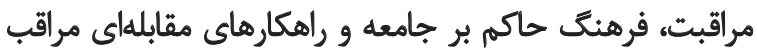

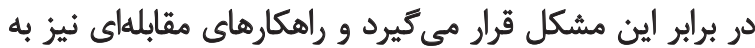

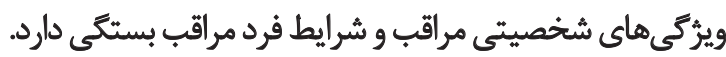
مطالعات نشان مىدهند كه رفتارهاى مقابلهاى نظير افزايش

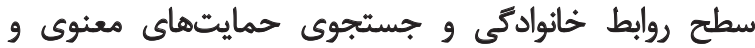

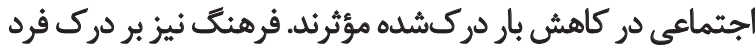

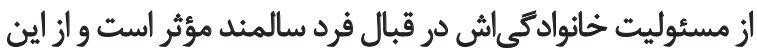

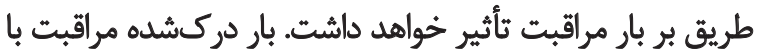

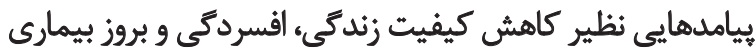

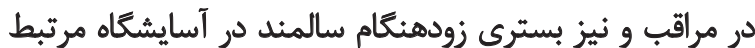

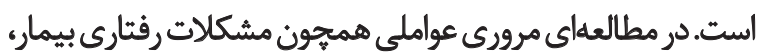

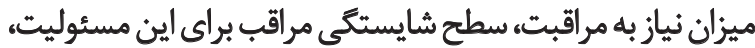

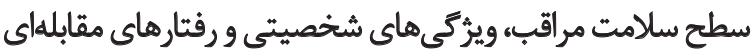

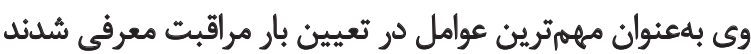
[ب]

محققان بار دركشده ذهنى را بهعنوان ييش كويى كنئدهاي

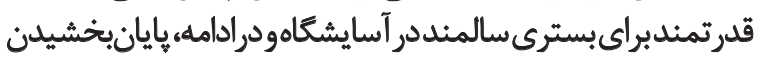

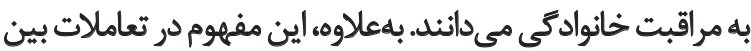

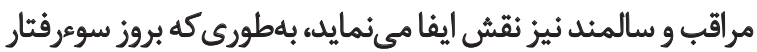

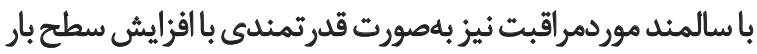

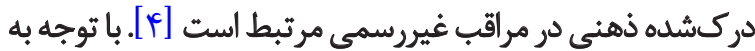

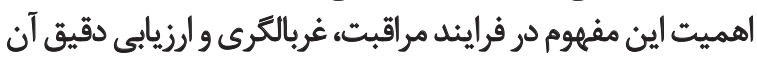
و طراحى مداخلات بههنكام براى كاهش آن ضرورى است.

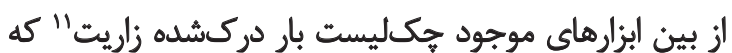

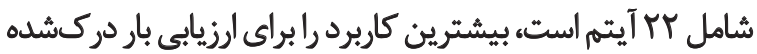

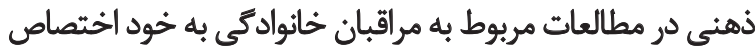

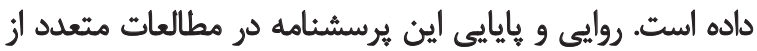

10. Subjective \& objective burden

11. Zarit Burden Interview (ZBI)
و اسكويوس با كليدواثههاي -Appraisal, Caregiving, Fam ily caregiving, burden, Satisfaction, Positive aspect, Negative aspect, Older adults, Elderly اطلاعاتى فارسىزبان بإيخاه اطلاعات علمى جهاد

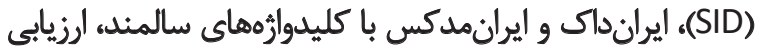
مراقبت، مراقبت خانوادكى، بار مراقبت و فشار مراقبت انجام كرفتئه أست.

در اين جستجو محدوديت زمانى درنظرترفته نشد و فرايند

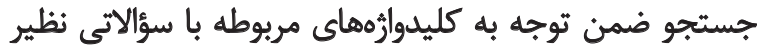

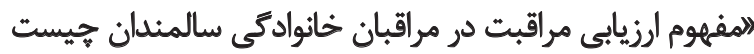

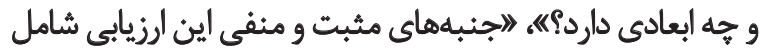

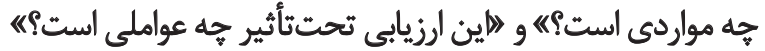

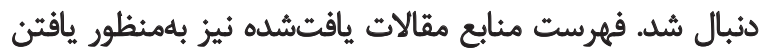
مقالات مرتبط موردبررسى قرار كرفت.

براى شروع، عنوان و خكيده مقالات مطالعه و مقالات براى مرور

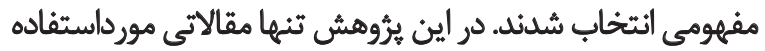

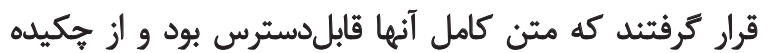

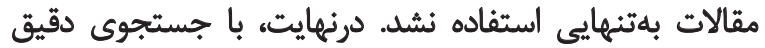

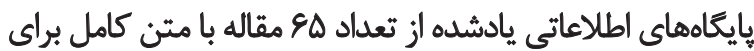
اين مرور استفاده شدا شئد

يافتها

درادامه، يافتههاى حاصل از مرور مطالعات در حوزه مراقبان

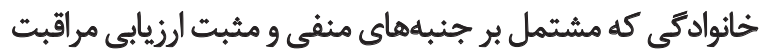

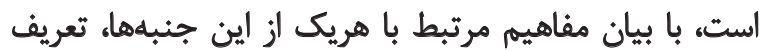

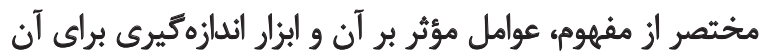

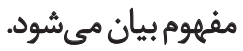

$$
\begin{aligned}
& \text { الف. جئبهانى مئفى الرزيابي مراقبت } \\
& \text { بارمراقبتv }
\end{aligned}
$$

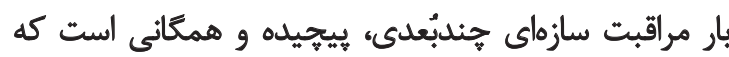

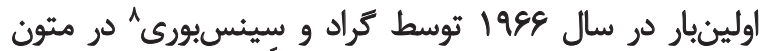

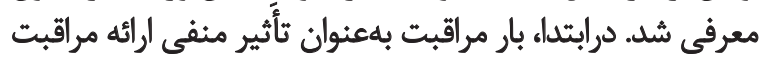

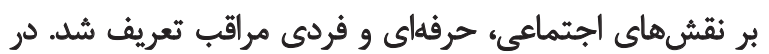

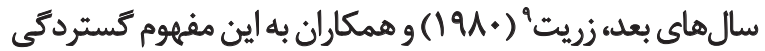

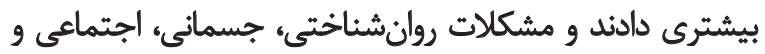

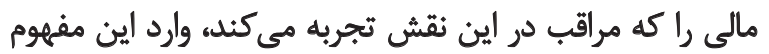

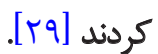

\footnotetext{
7. Caregiving burden

8. Grad and Sainsbury

9. Zarit
} 


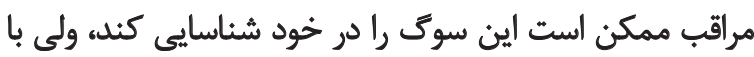

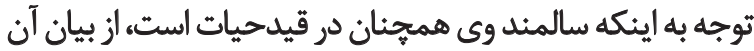
احساس شرمسارى نمايد.

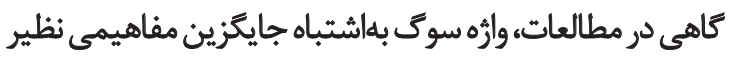

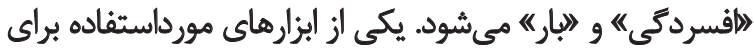

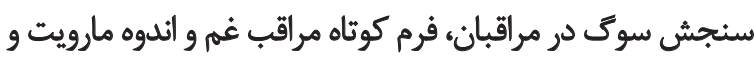

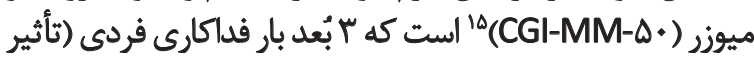

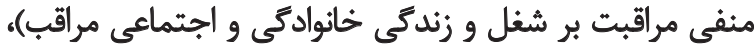

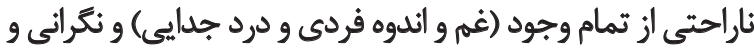

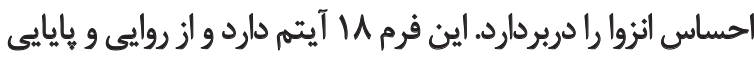

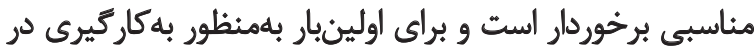

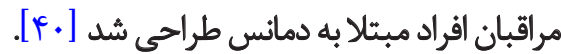

$$
\text { فشار مراقبت }
$$

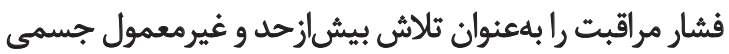

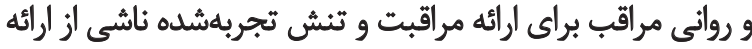

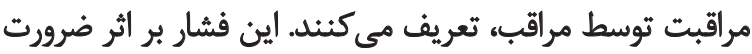

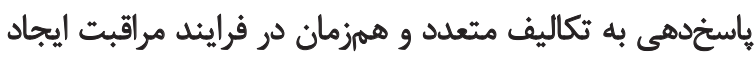
ميشود و به احساس سختى كار در حين ايفاى نقش مراقيت مرايت

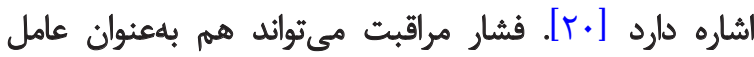

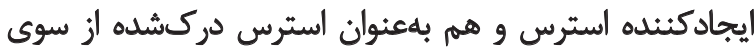
مراقبت تعريف شود.

ازآنجايىكه افزايش فشار مراقب با بسترىشدن زودهنكام

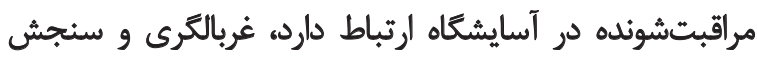

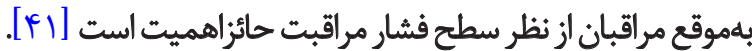

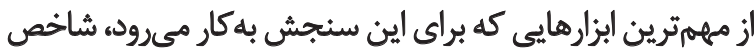

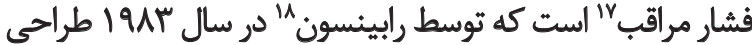

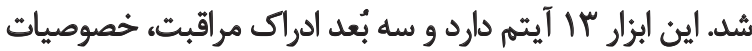

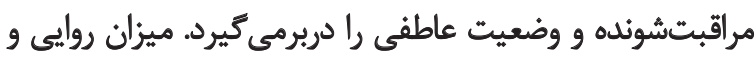

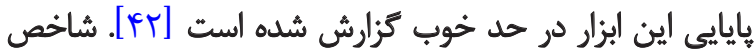

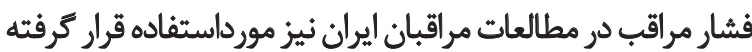

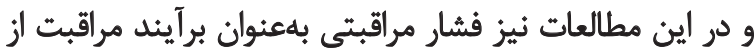

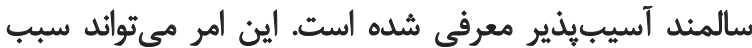

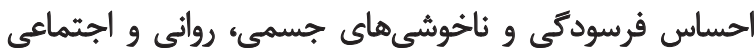

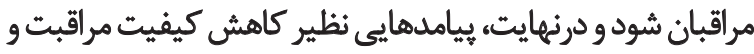

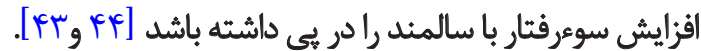

15. Marwit and Meuser Caregiver Grief Inventory (MM-CGI-50)

16. Caregiving strain

17. Caregiver strain index

18. Robinson

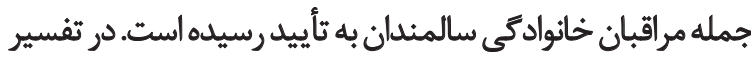

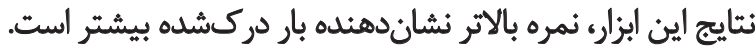

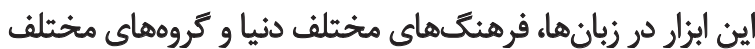

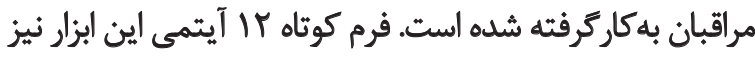

$$
\text { سودسترس است [Tr]. }
$$

سوك يكى از اجزاى اصلى تجربه مراقبان غيررسمى، بهويره

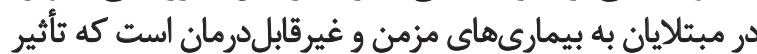

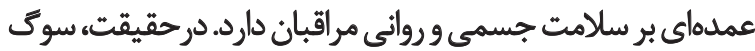

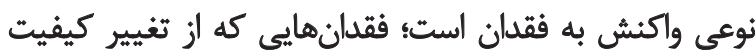

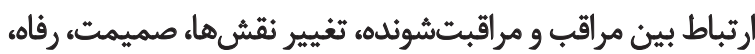

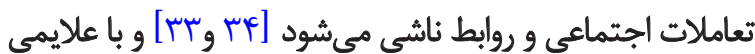

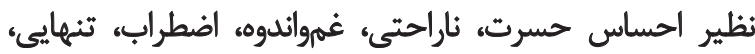

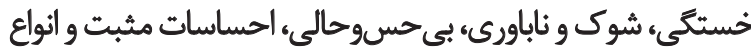

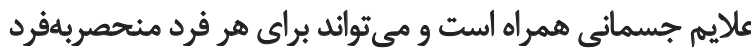

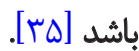

سوك زمانى خخ ميدهد كه فرد امنيت و اطمينانى را كه در

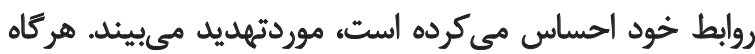

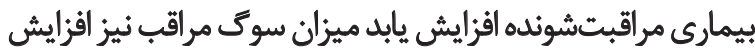

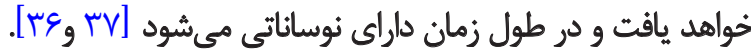

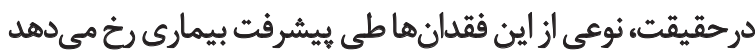

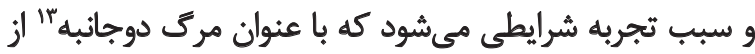

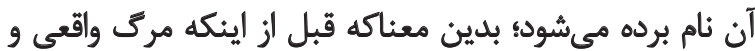

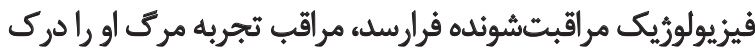

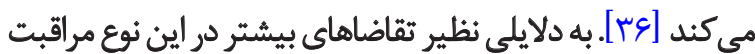

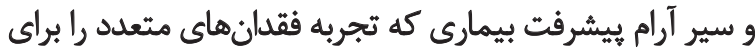

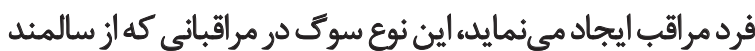
مبتلا به دمانس مراقبت مى كنيند نسبت به ديكر مراقيت مراقبان بيشتر

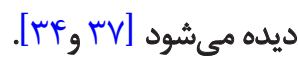

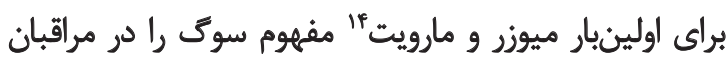

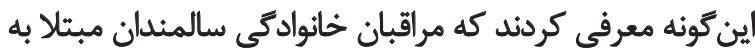

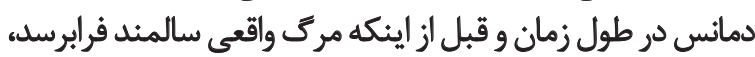

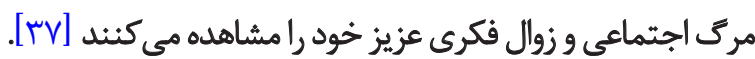

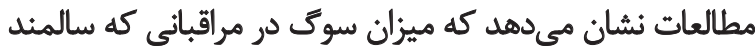

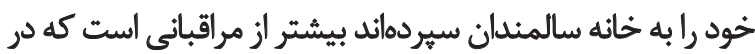

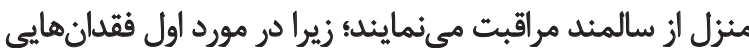

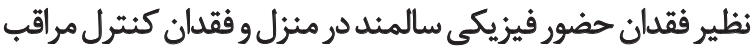

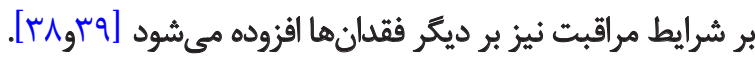

12. Grief

13. Dual dying

14. Meuser and Marwit 
غيرعاطفى نظير انجام بهترين كار در مراقبت به رضايتمندى

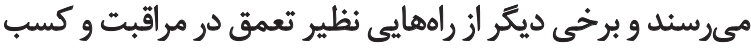

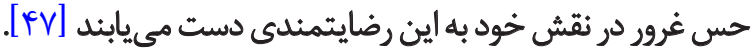

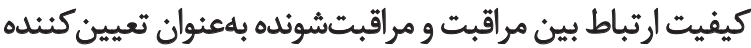

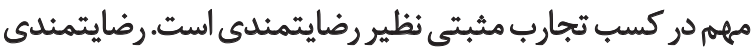

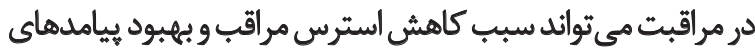

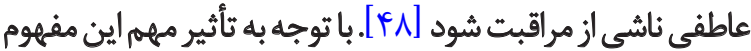

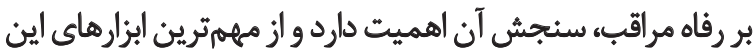

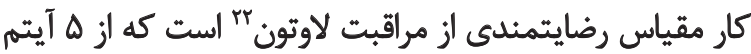

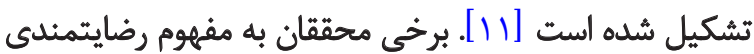

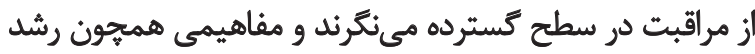
فردى، عمل متقابل و.... رادر آن وارد كردهاند.

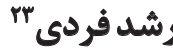

مفهوم رشد فردى اولينبار در متون مربوط به تروماو سوكى يس

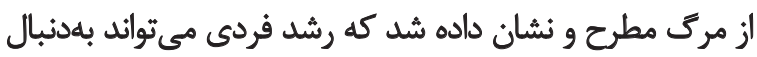

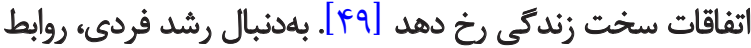

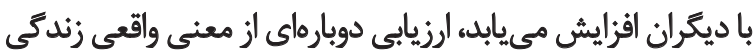

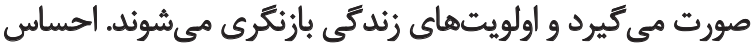

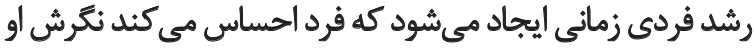

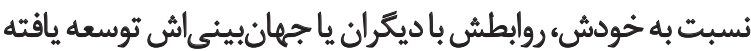

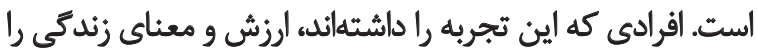

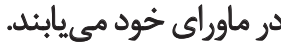

يديده رشد به افراد اجازه مى دهد تا باوجود سختى هائ موجودي،

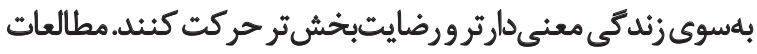

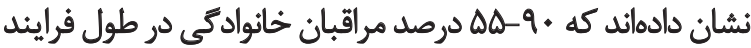

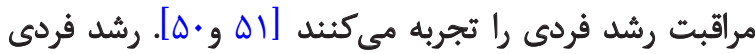

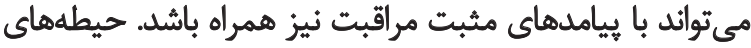

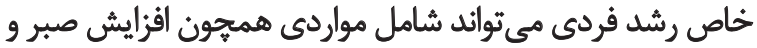

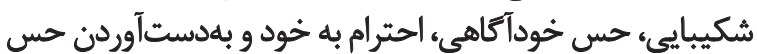

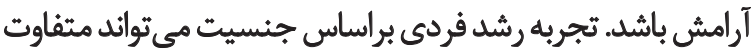

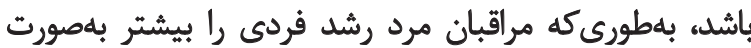
فروتنشدن نشان مي مهند [FV]

يكى از ابزارهايى كه در ارزيابى رشد فردى مراقبان خانوادكى

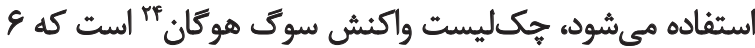

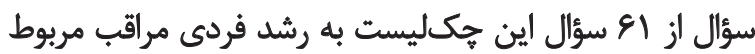

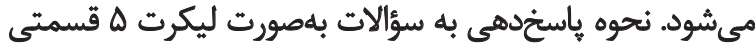

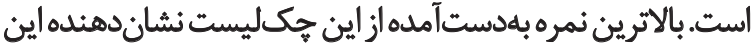

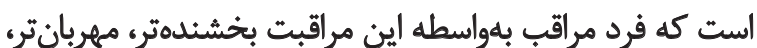

22. Caregiving satisfaction scale

23. Personal growth

24. Hogan Grief Reaction Checklist

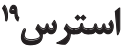

استرس بهنوعى عدمتعادل شناختى بين تثاضاى دركشده

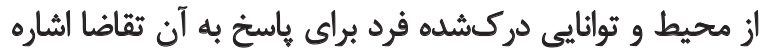

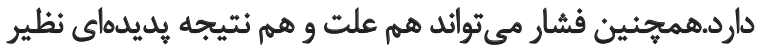

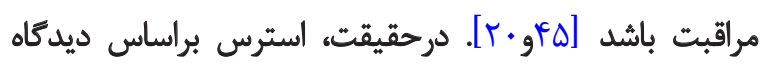

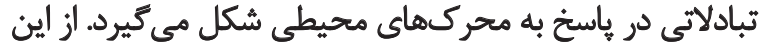

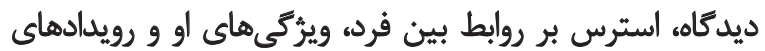

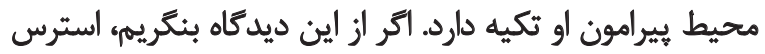

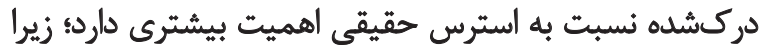

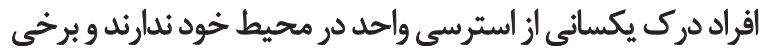
آن راكمتر و برخى بيشتر از حد واقعى درك كنيند.

فرايند استرس و مقابله باآن، در الكوهاي معروفي مورد كنكاش

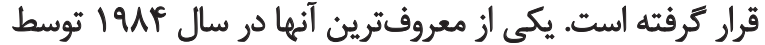

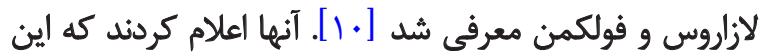

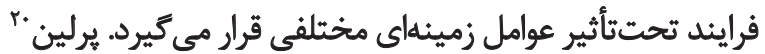

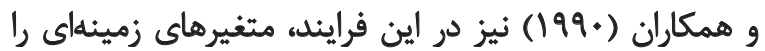

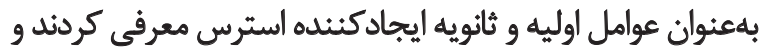

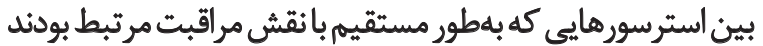

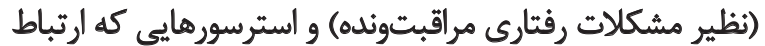
غيرمستقيم داشتند (نظير مشكلات مالى مراقب) تمايز قائل شدند النداط

[rV]

\section{ب. جنبههاي مشبت ارزيابي مراقبت}

\section{رضايتمندى ازمراقبت"}

مفهوم رضايتمندى از مراقبت، يكى از معمولترين مفاهيم

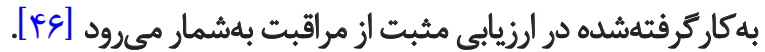

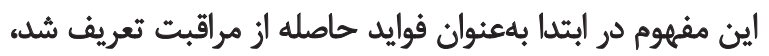

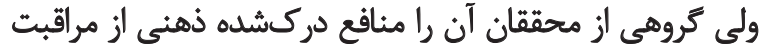

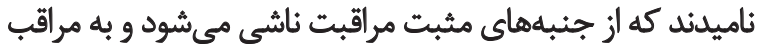

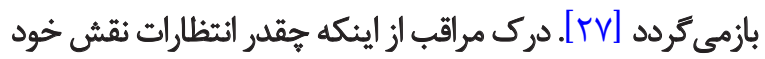

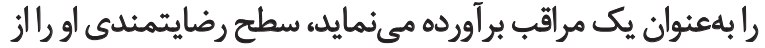

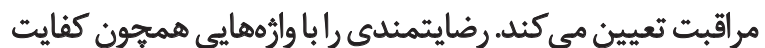

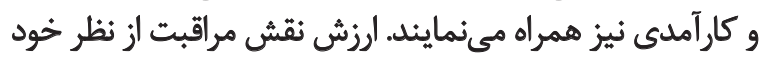

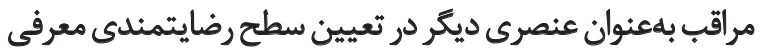

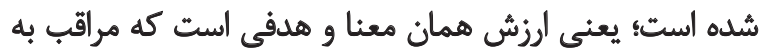

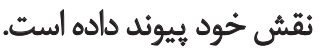

مطالعات نشان داده است كه كيفيت اين رضايت در مراقبان مختلف متفاوت است، بلطورىكه برخى مراقبان از راههاي

21. Caregiving satisfaction 
مراقبت" است كه توسط فارن" و همكارانش در سال 1999

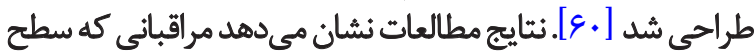

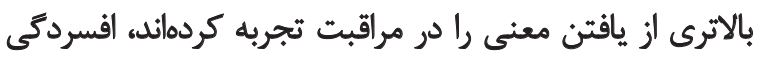

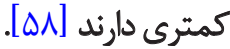

\section{احساسموفقيت}

احساس موفقيت يكى از ابعاد جنبههاى مثبت تجارب مراقبت

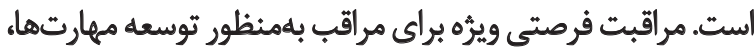

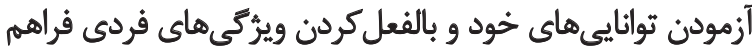

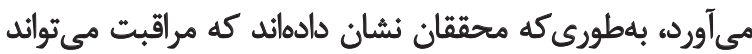
احساس موفقيت و غرور را در فرد مراقب ايجاد كند [إعان و و

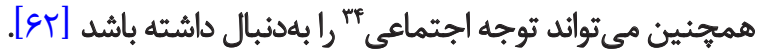

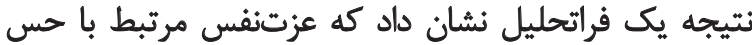

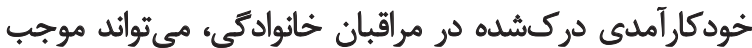

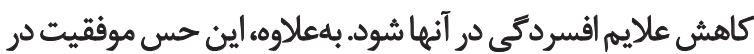

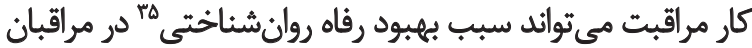

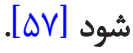

محققان بسيارى به حس موفقيت در كار برداخته و از آن آن

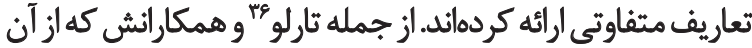

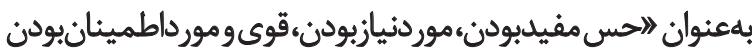

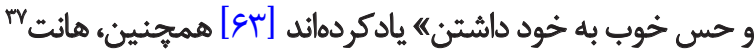

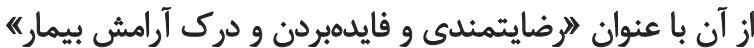

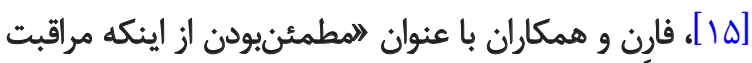

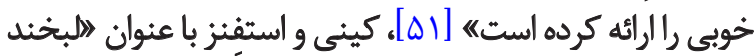

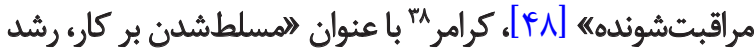

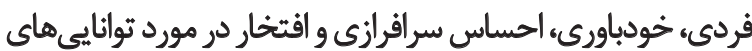

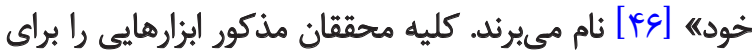

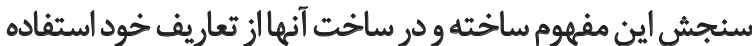

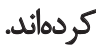

\section{تسلط بر كار مراقبت}

تسلط بر مراقبت، درى افراد از توانايعشان براى كنترل نيروهايى كه زندكى آنها راتحت تأثير قرار مي دهده، تعريف شده

31. Finding meaning through caregiving scale

32. Farren

33. Feeling of accomplishment

34. Social recognition

35. Psychological well-being

36. Tarlow

37. Hunt

38. Kramer

39. Caregiving mastery
صبورتر، اميدوارتر و دلسوزتر شده است. ضريب آلفاى كرونباخ اين

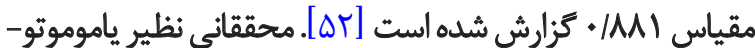

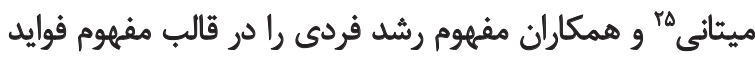

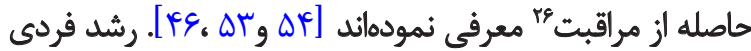

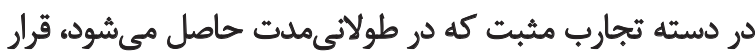

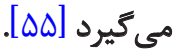

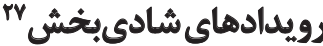

اولينبار كينى و استفنز مَ تجارب مثبت در مراقبت را بهعنوان

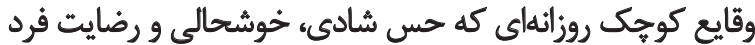

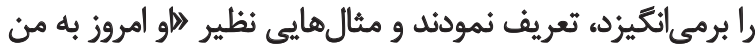

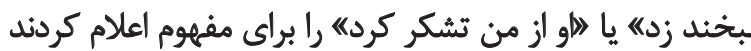

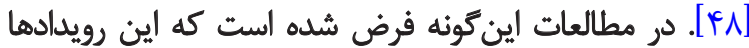

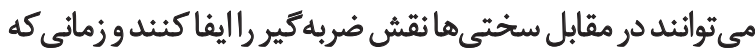

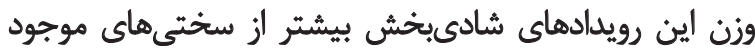

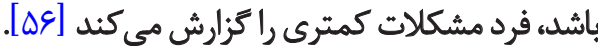

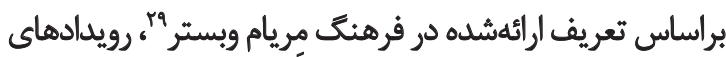
شادىبخش موجب بهبود شرايط معنوى، اجتماعي و فكرى أفراد

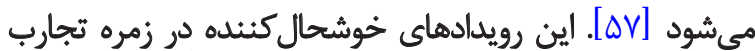

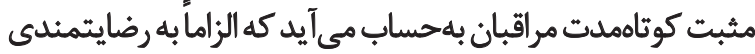

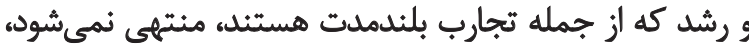

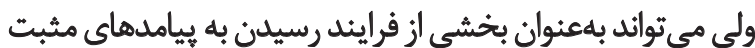

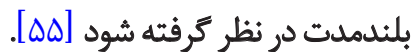

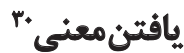
اين سازه از طريق مطالعات كيفى شناسايى شد. درحقيقت،

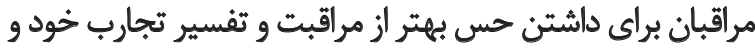

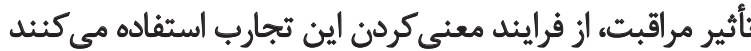

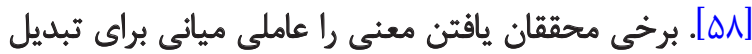

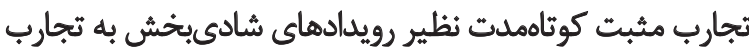

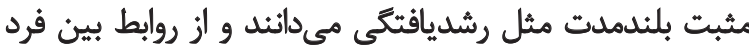

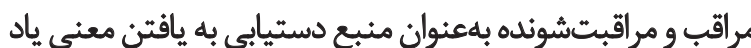

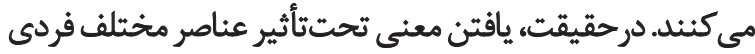
نظير شخصيت، جهانبينى، حمايت اجتماعي، وضعيت اجتئيت إتماعى

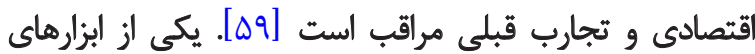

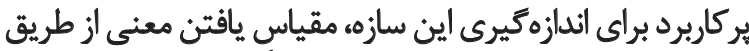

25. Yamamoto-Mitan

26. Consequential gain

27. Uplift

28. Kinney and Stephens

29. Merriam Webster

30. Finding meaning 
معنوى، ياداشهاي عاطفى و حس شايستّى و تسلط بر كار

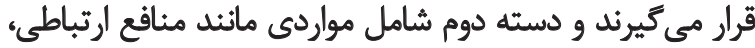

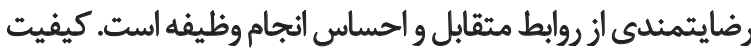

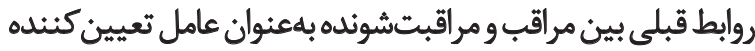

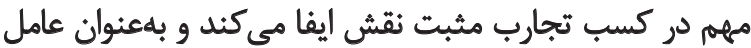

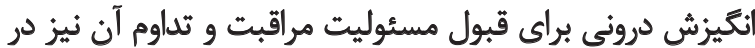

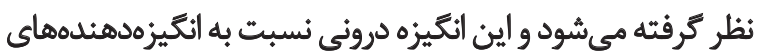

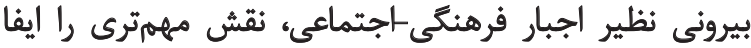

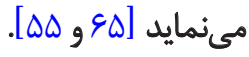
باوجود ظرفيت بالقوه موجود در جنبههاى مثبت مراقبت براى

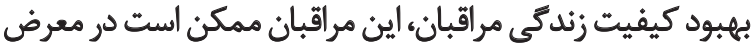

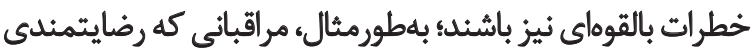

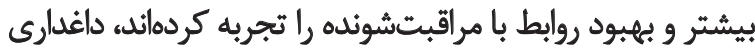

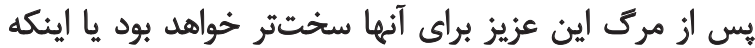

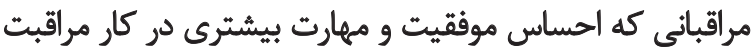

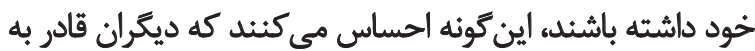

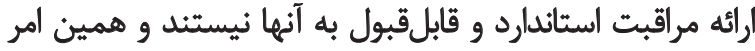

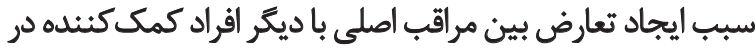

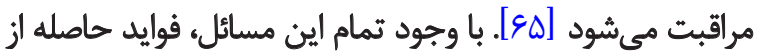

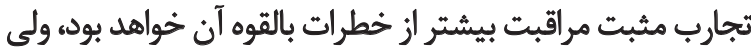

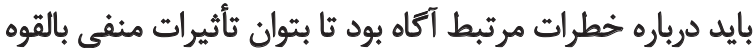

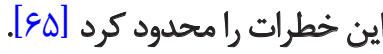

در تمامى جنبههاى منفى دركشده از مراقبت، بهنوعى برافي

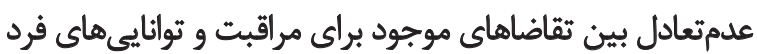

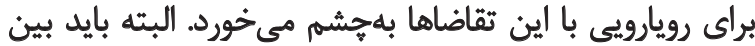

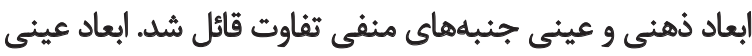

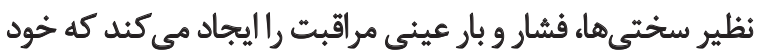

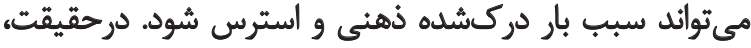

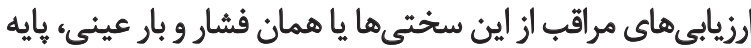

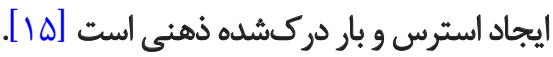

براساس مرور انجامخرفته بر متون موجود، استرس و بار مراقبت

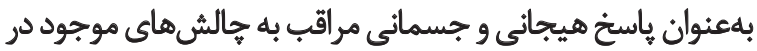

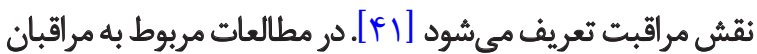
ديده مىشود كه وارمهاى استرس، فشار مراقبت و وبار مراقبت

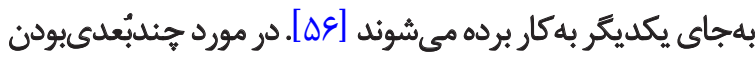

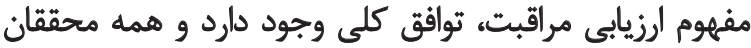

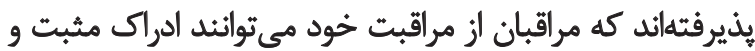

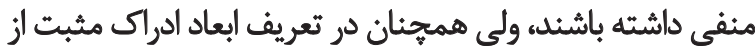

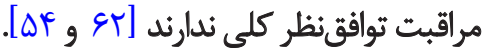

در مطالعات مختلف و ابزارهاى بهكارفته براى سنجش

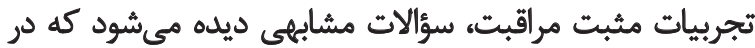

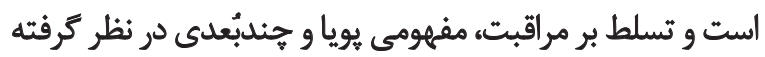

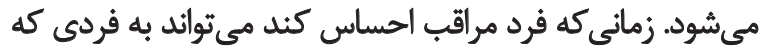

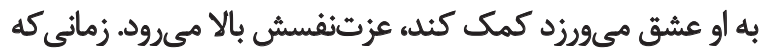

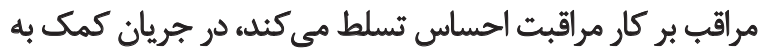

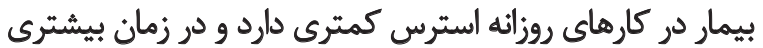

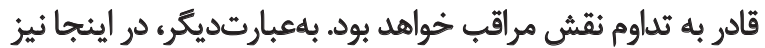

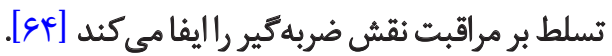
احساس مهارت يا شايستّى در انجام مراقبت در برخى مراقبان

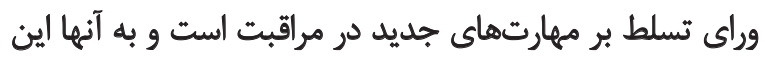

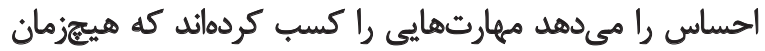

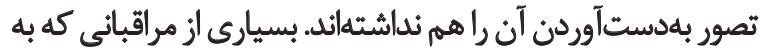

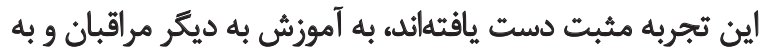

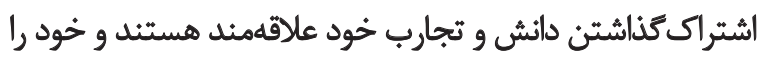
بلهنوان فردى حرفهاي در كار مراقبت تصور مى كنيند.

\section{بحث}

با توجه به رشد جمعيت سالمندى و نياز افراد ناتوان به مراقبت

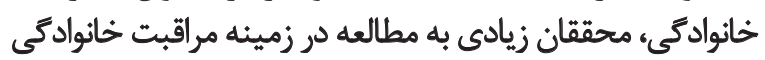

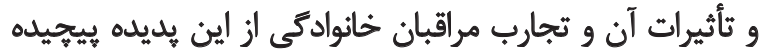

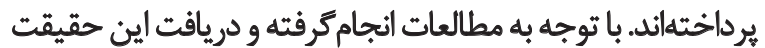
كه با وجود مشكلات فراوان در شرايط مراقبت، همه مراقبان ائن اين

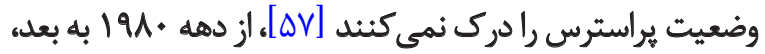

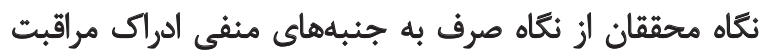

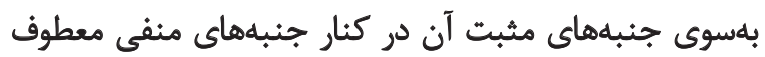

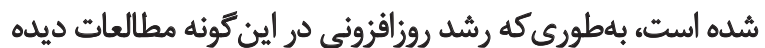

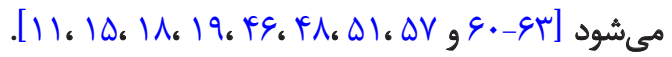
يافتههاى اوليهاى درباره جنبههاى مثبت مراقبت از مطالعات

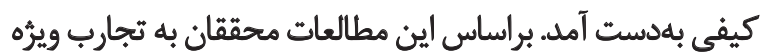

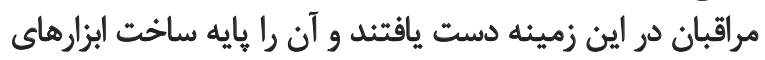

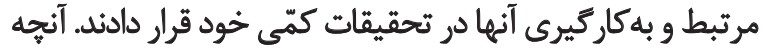

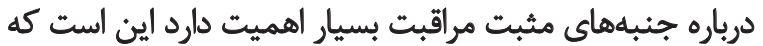

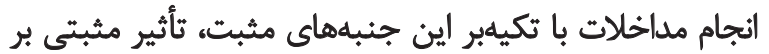

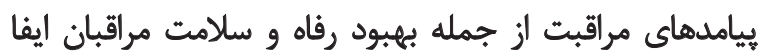

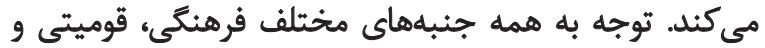

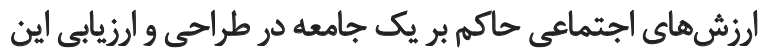

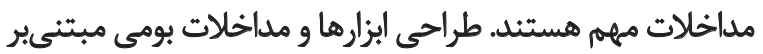

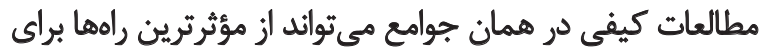
ارتقاى سلامت مراقبان و كيفيت مراقبت باشئ ماند.

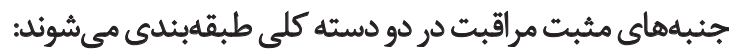

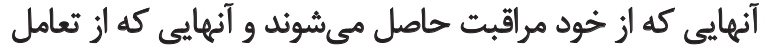

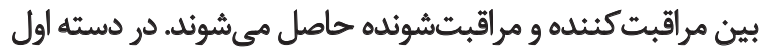

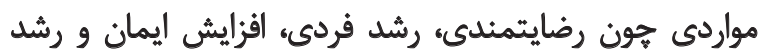




\section{References}

[1] United Nations Fund Population Agency (UNFPA). Ageing in the twenty-first century: A celebration and a challenge [Internet] New York: UNFPA and Help Age International; 2012. Available from: http://www.unfpa.org/publications/ageing-twenty-firstcentury.

[2] Kalaria RN, Maestre GE, Arizaga R, Friedland RP, Galasko D, Hall K, et al. Alzheimer's disease and vascular dementia in developing countries: Prevalence, management, and risk factors. Lancet Neurology. 2008; 7(9):812-26.

[3] Noroozian M. The elderly population in Iran: An ever growing concern in the health system. Iranian Journal of Psychiatry and Behavioral Sciences. 2012; 6(2):1-6.

[4] Given CW, Given BA, Stommel M, Azzouz F. The impact of new demands for assistance on caregiver depression: Tests using an inception cohort. Gerontologist. 1999; 39(1):76-85.

[5] Volpin M. The experiences of family caregivers of assisted living residents enrolled in Hospice. Journal of Pain and Symptom Management. 2010; 39(2):344-5

[6] Alice Lau WY, Lotus Shyu YI, Lin LC, Yang PS. Institutionalized elders with dementia: Collaboration between family caregivers and nursing home staff in Taiwan. Journal of Clinical Nursing. 2008; $17(4): 482-90$

[7] Papastavrou E, Kalokerinou A, Papacostas SS, Tsangari H, Sourtzi P. Caring for a relative with dementia: Family caregiver burden. Journal of Advanced Nursing. 2007; 58(5):446-57.

[8] Annerstedt L, ElmstÅhl S, Ingvad B, Samuelsson SM. An analysis of the caregiver's burden and the "breaking-point" when home care becomes inadequate. Scandinavian Journal of Public Health. 2000; 28(1):23-31.

[9] Walker AJ, Pratt CC, Eddy L. Informal caregiving to aging family members: A critical review. Family Relations. 1995; 44(4):402-11.

[10] Lazarus RS, Folkman S. Stress, appraisal, and coping. New York: Springer Publishing Company; 1984.

[11] Lawton MP, Kleban MH, Moss M, Rovine M, Glicksman A. Measuring caregiving appraisal. Gerontology. 1989; 44(3):61-71.

[12] Aranda SK, Hayman-White K. Home caregivers of the person with advanced cancer: An Australian perspective. Cancer Nursing. 2001; 24(4):300-7.

[13] Gilbert P, Bhundia R, Mitra R, McEwan K, Irons C, Sanghera J. Cultural differences in shame-focused attitudes towards mental health problems in Asian and non-Asian student women. Mental Health, Religion \& Culture. 2007; 10(2):127-41.

[14] Wong MYF, Chan SWC. The qualitative experience of Chinese parents with children diagnosed of cancer. Journal of Clinical Nursing. 2006; 15(6):710-7.

[15] Hunt CK. Concepts in caregiver research. Journal of Nursing Scholarship. 2003; 35(1):27-32.

[16] Braithwaite V. Between stressors and outcomes: Can we simplify caregiving process variables? Gerontologist. 1996; 36(1):42-53.

[17] Abdulraheem I. An opinion survey of caregivers concerning caring for the elderly in Ilorin metropolis, Nigeria. Public Health. 2005; 119(12):1138-44.
معاني متفاوت و ابعاد محتثلف قرار كرفتهاند. اين موضوع ثأييدي

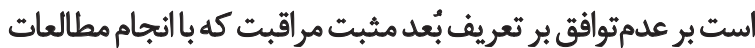

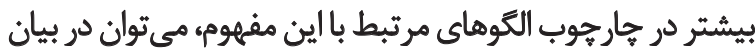
تعريف جنبه مثبت مراقبت به توافق بيشتر دست يافت. با توجيه

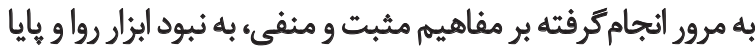

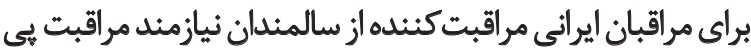

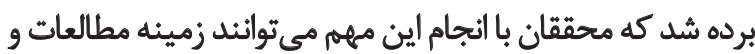

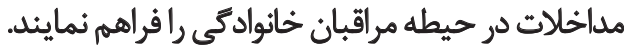

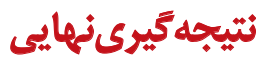

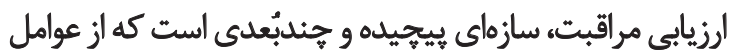

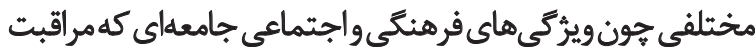

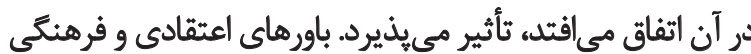

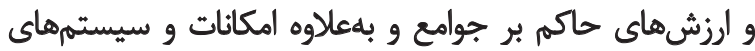

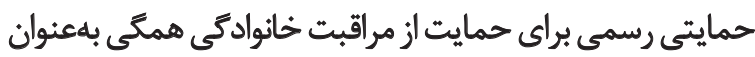

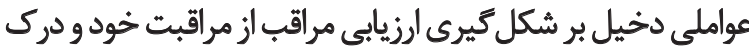
وى از اين نوع مراقبت و يهيامدهاي آن مؤثر است.

با توجه به تفاوتهاى فرهنكى و اجتماعى در جامعه ايرانى در

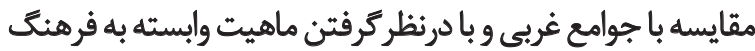

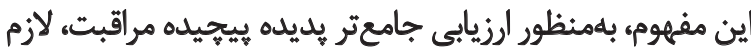

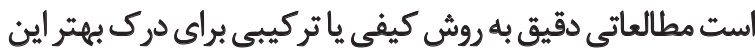

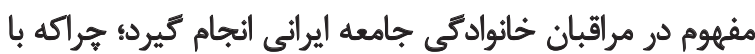

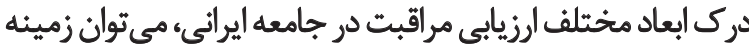

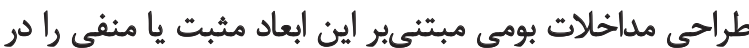
مراقبان خانوادكي سالمندان فراهي ماهم نمود.

$$
\text { تشيكر وقدرداني }
$$

اين مقاله قسمتى از رساله اكرم فرهادى دانشجوى دكتراى

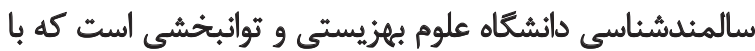

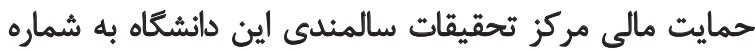

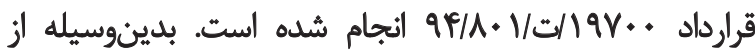

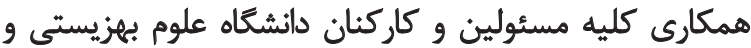
توانبخشي كمال تشكر و قدرداني بهعمل مي مئيد 
[18] Chao SY, Roth P. The experiences of Taiwanese women caring for parents-in-law. Journal of Advanced Nursing. 2000; 31(3):631-8.

[19] Gates KM. The experience of caring for a loved one: A phenomenological study. Nursing Science Quarterly. 2000; 13(1):54-9.

[20] Kristensson Ekwall A. Informal caregiving at old age. Content, coping, difficulties and satisfaction [PhD dissertation]. Sweden: Lund University; 2004.

[21] Shaibu S, Wallhagen MI. Family caregiving of the elderly in Botswana: Boundaries of culturally acceptable options and resources. Journal of Cross-Cultural Gerontology. 2002; 17(2):139-54

[22] Subgranon R, Lund DA. Maintaining caregiving at home: A culturally sensitive grounded theory of providing care in Thailand. Journal of Transcultural Nursing. 2000; 11(3):166-73.

[23] McCleary L, Blain J. Cultural values and family caregiving for persons with dementia. Indian Journal of Gerontology. 2013; 27(1):178-201.

[24] Morano CL. Appraisal and coping: Moderators or mediators of stress in Alzheimer's disease caregivers? Social Work Research. 2003; 27(2):116-28.

[25] Morano CL. The role of appraisal and expressive support in mediating strain and gain in Hispanic Alzheimer's disease caregivers. Journal of Ethnic and Cultural Diversity in Social Work. 2003; 12(2):1-18.

[26] Harwood DG, Barker WW, Ownby RL, Bravo M, Aguero H, Duara R. Predictors of positive and negative appraisal among $\mathrm{Cu}$ ban American caregivers of Alzheimer's disease patients. International Journal of Geriatric Psychiatry. 2000; 15(6):481-7.

[27] Pearlin LI, Mullan JT, Semple SJ, Skaff MM. Caregiving and the stress process: An overview of concepts and their measures. Gerontologist. 1990; 30(5):583-94.

[28] Lundh U. Family carers 2: sources of satisfaction among Swedish carers. British Journal of Nursing. 1999; 8(10):647-52.

[29] Zarit SH, Reever KE, Bach-Peterson J. Relatives of the impaired elderly: Correlates of feelings of burden. Gerontologist. 1980; 20(6):649-55

[30] Chou KR. Caregiver burden: a concept analysis. Journal of Pediatric Nursing. 2000; 15(6):398-407.

[31] van der Lee J, Bakker TJ, Duivenvoorden HJ, Dröes RM. Multivariate models of subjective caregiver burden in dementia: A systematic review. Ageing Research Reviews. 2014; 15:76-93.

[32] Zarit SH, Todd PA, Zarit JM. Subjective burden of husbands and wives as caregivers: A longitudinal study. Gerontologist. 1986; 26(3):260-6.

[33] Loos C, Bowd A. Caregivers of persons with Alzheimer's disease: Some neglected implications of the experience of personal loss and grief. Death Studies. 1997; 21(5):501-14.

[34] Sanders S, Corley CS. Are they grieving? A qualitative analysis examining grief in caregivers of individuals with Alzheimer's disease. Social Work in Health Care. 2003; 37(3):35-53.

[35] Rando TA. Clinical dimensions of anticipatory mourning: Theory and practice in working with the dying, their loved ones, and their caregivers. Champaign, I.L.: Research Press; 2000.

[36] Ott CH, Sanders S, Kelber ST. Grief and personal growth experience of spouses and adult-child caregivers of individuals with Alzheimer's disease and related dementias. Gerontologist. 2007; 47(6):798-809.
[37] Meuser TM, Marwit SJ. A comprehensive, stage-sensitive model of grief in dementia caregiving. Gerontologist. 2001; 41(5):658-70

[38] Miniño AM, Murphy SL, Xu J, Kochanek KD. Deaths: Final data for 2008. National vital statistics Reports. 2011; 59(10):1-26.

[39] Rudd MG, Viney LL, Preston CA. The grief experienced by spousal caregivers of dementia patients: The role of place of care of patient and gender of caregiver. International Journal of Aging and Human Development. 1999; 48(3):217-40.

[40] Marwit SJ, Meuser TM. Development of a short form inventory to assess grief in caregivers of dementia patients. Death Studies. 2005; 29(3):191-205.

[41] Thornton M, Travis SS. Analysis of the reliability of the modified caregiver strain index. Journals of Gerontology Series B: Psychological Sciences and Social Sciences. 2003; 58(2):127-32.

[42] Robinson BC. Validation of a caregiver strain index. Gerontology. 1983; 38(3):344-8.

[43] Mohammadi F, Babaee M. [Effects of participation in support groups on Alzheimer's family caregivers' strain and spiritual wellbeing (Persian)]. Iranian Journal of Ageing. 2011; 6(1):29-37.

[44] Mohammadi F, Kaldi AR. [Self efficacy and caregiver strain in Alzheimer's caregivers (Persian)]. Iranian Journal of Ageing. 2006; 3(3):8-12

[45] Nolan MR, Grant G, Ellis NC. Stress is in the eye of the beholder: reconceptualizing the measurement of carer burden. Journal of Advanced Nursing. 1990; 15(5):544-55.

[46] Kramer BJ. Gain in the caregiving experience: Where are we? What next? Gerontologist. 1997; 37(2):218-32.

[47] Lloyd J, Patterson T, Muers J. The positive aspects of caregiving in dementia: A critical review of the qualitative literature. Dementia. 2014: 1471301214564792 [Epub ahead of print].

[48] Kinney JM, Stephens MA. Hassles and uplifts of giving care to a family member with dementia. Psychology and Ageing. 1989; $4(4): 402-8$.

[49] Hogan NS, Schmidt LA. Testing the grief to personal growth model using structural equation modeling. Death Studies. 2002; 26(8):615-34.

[50] Butcher HK, Holkup PA, Buckwalter KC. The experience of caring for a family member with Alzheimer's disease. Western Journal of Nursing Research. 2001; 23(1):33-55.

[51] Farran CJ, Keane-Hagerty E, Salloway S, Kupferer S, Wilken CS. Finding meaning: An alternative paradigm for Alzheimer's disease family caregivers. Gerontologist. 1991; 31(4):483-9.

[52] Hogan S, Daryl B, Lee A, Schmidt N. Development and validation of the Hogan grief reaction checklist. Death Studies. 2001; 25(1):1-32.

[53] Yamamoto-Mitani N, Sugishita C, Ishigaki K, Hasegawa K, Maekawa N, Kuniyoshi M, et al. Development of instruments to measure appraisal of care among Japanese family caregivers of the elderly. Research and Theory for Nursing Practice. 2001; 15(2):113-35

[54] Kate N, Grover S, Kulhara P, Nehra R. Scale for positive aspects of caregiving experience: development, reliability, and factor structure. East Asian Archive of Psychiatry. 2012; 22(2):62-9.

[55] Siegel K, Schrimshaw EW, Pretter S. Stress-related growth among women living with HIV/AIDS: Examination of an 
explanatory model. Journal of Behavioral Medicine. 2005; 28(5):403-14.

[56] Kinney JM, Stephens MAP, Franks MM, Norris VK. Stresses and satisfactions of family caregivers to older stroke patients. Journal of Applied Gerontology. 1995; 14(1):3-21.

[57] Pinquart M, Sörensen S. Associations of caregiver stressors and uplifts with subjective well-being and depressive mood: A metaanalytic comparison. Aging \& Mental Health. 2004; 8(5):438-49.

[58] Farran CJ, Miller BH, Kaufman JE, Davis L. Race, finding meaning, and caregiver distress. Journal of Aging Health. 1997; 9(3):316-33.

[59] Park CL. Stress-related growth and thriving through coping: The roles of personality and cognitive processes. Journal of Social Issues. 1998; 54(2):267-77.

[60] Farran CJ, Miller BH, Kaufman JE, Donner E, Fogg L. Finding meaning through caregiving: Development of an instrument for family caregivers of persons with Alzheimer's disease. Journal of Clinical Psychology. 1999; 55(9):1107-25.

[61] Noonan AE, Tennstedt SL, Rebelsky FG. Making the best of it: Themes of meaning among informal caregivers to the elderly. Journal of Ageing Studies. 1997; 10(4):313-27.

[62] Motenko AK. The frustrations, gratifications, and well-being of dementia caregivers. Gerontologist. 1989; 29(2):166-72.

[63] Tarlow BJ, Wisniewski SR, Belle SH, Rubert M, Ory MG, Gallagher-Thompson D. Positive aspects of caregiving contributions of the REACH Project to the development of new measures for Alzheimer's caregiving. Research on Ageing. 2004; 26(4):429-53.

[64] Upton D, Upton P, Alexander R. The complexity of caring (Part 2): Moderators of detrimental health and well-being outcomes for caregivers of people with chronic wounds. Wound Practice \& Research: Journal of the Australian Wound Management Association. 2015; 23(3):110-114

[65] Carbonneau H, Caron C, Desrosiers J. Development of a conceptual framework of positive aspects of caregiving in dementia. Dementia. 2010; 9(3):327-53. 
